\title{
Fuzzy Robust Design of Dynamic Vibration Absorbers
}

\author{
A. D. G. Silva, A. A. Cavalini Jr, and V. Steffen Jr \\ Laboratory of Mechanics and Structures (LMEst), School of Mechanical Engineering, Federal University of Uberlândia, \\ Avenida João Naves de Ávila 2121, 38408-196 Uberlândia, MG, Brazil \\ Correspondence should be addressed to V. Steffen Jr; vsteffen@mecanica.ufu.br
}

Received 8 October 2015; Accepted 7 December 2015

Academic Editor: Chao Tao

Copyright @ 2016 A. D. G. Silva et al. This is an open access article distributed under the Creative Commons Attribution License, which permits unrestricted use, distribution, and reproduction in any medium, provided the original work is properly cited.

\begin{abstract}
This paper is dedicated to the development of robust optimization and decision making techniques taking into account the uncertain parameters of linear and nonlinear dynamic vibration absorbers. In this case, novel approaches are proposed regarding different fuzzy logic optimization strategies. The uncertain parameters of the considered mechanical systems are treated as fuzzy variables. Consequently, the associated optimization problem is described as a fuzzy function that maps the fuzzy inputs. The proposed techniques are applied to the design of dynamic vibration absorbers. This numerical study illustrates the versatility and convenience of the proposed fuzzy logic optimization strategies.
\end{abstract}

\section{Introduction}

The design process and operation limits of mechanical systems are currently obtained from the analysis of deterministic models, which should be able to represent the associated dynamic phenomena. However, parametric or nonparametric uncertainties are inherent to dynamic systems due to manufacturing errors and tolerances, damage, wear, and influence of environmental conditions that should be taken into account to obtain reliable mathematical models. These fluctuations are disregarded in ordinary deterministic approaches. In this sense, deterministic models can be considered inaccurate and should be replaced by models that take into account the uncertainties.

There are innumerous approaches for modeling uncertainties in mechanical systems. Among them, both the stochastic and fuzzy logic approaches are widely used. The fuzzy logic [1] is an intuitive approach that takes into account associated fuzzy sets and the possibility theory [2], while the stochastic methodology is based on the probability theory [3-5]. Both methodologies have been successfully applied to various problems in engineering, leading to similar results. The stochastic approach shows a longer history of applications to mechanical systems [6-10] and, consequently, seems to be more established than the fuzzy logic. However, the fuzzy approach is receiving more attention in our days and the literature brings some successful applications [11-14].

The optimum design of a given mechanical system must be robust with respect to unavoidable uncertainties that affect its dynamic behavior. Regarding the optimization issues, robustness can be understood as a design that is frequently not the best solution obtained for a particular dynamic problem. However, the robust solution is a better solution for the cases in which the system is exposed to parameter fluctuations. Therefore, a robust design can be considered as the one wherein the goal is to obtain the best solution for a given uncertain scenario. The uncertainty analysis is associated with an optimization procedure in order to determine the robust design for the system (i.e., the robust optimization procedure).

Taguchi [15] is the pioneer in the area of robust optimization. He proposed the insertion of noise factors in the cost functions, thus defining a mean square deviation that has to be maximized in terms of the design variables. Commonly, the robust optimization approaches rely on stochastic techniques based on the statistical moments of the problem [16-21] and in the definition of a robust objective function. This approach leads to large combinatorial multiobjective problems, imposing a serious disadvantage regarding the associated computational cost. The fuzzy logic is a simpler 
mathematical logic for uncertainty analysis, as based on pessimistic and optimistic values (i.e., fuzzy sets). Therefore, the fuzzy logic seems to be well suited for robust optimization.

In this context, the present contribution aims at proposing different fuzzy robust optimization techniques. These methodologies were evaluated for the design of linear and nonlinear robust dynamic vibration absorbers (DVA and nDVA, resp.), considering parametric uncertainties affecting the mechanical system. The uncertainties are treated as fuzzy variables so that the optimization model becomes a fuzzy function that maps the fuzzy inputs. Therefore, a fuzzy evaluation of the optimization problem can be conceived through the evaluation of the pessimistic and optimistic values of the fuzzy sets (i.e., the uncertain information). This is the basic concept of the techniques conveyed in the present contribution.

This paper is organized as follows. First, the key concepts of fuzzy logic are discussed; then, the fuzzy robust optimization techniques are presented. In the sequence, two design problems are described and the robust optimization results are discussed. Finally, a few remarks are made and further research works are proposed.

\section{Fuzzy Logic}

Fuzzy logic is the term used for the intuitive formulation proposed by Zadeh [1] that generalizes the membership notion of an element in a mathematical set, creating a graded notion represented by a membership function. In a classical sense, a math set is the collection of elements that belongs to a given definition. Any element can belong or not to the mentioned set. Regarding the fuzzy approach, the Boolean notion of the membership function is replaced by a graded one. Thus, an element can belong, not belong, or partially belong to the so-called fuzzy set.

As presented in [22], the membership function of a fuzzy set can be basically understood through three different perspectives, as follows: the degree of similarity, in which the membership function quantifies how similar an element of the set is, as compared to a prototype element; the degree of preference, where the membership function quantifies the preference or the feasibility of an element of the set; the degree of uncertainty, in which the membership function is the degree of possibility that a determined parameter $u$ assumes the value of a specific element $x$ of the set.

Based on the concept of degree of uncertainty, a fuzzy set can be understood as the union of the pessimistic and optimistic values of an uncertain parameter considering each level of possibility (i.e., $\alpha$-levels of the membership function). It is important to point out that the membership function is a possibility distribution, not a probability distribution as used in the case of the stochastic theory. Additionally, possibility is the measure of whether an event can happen, while probability is a measure of whether an event will happen. Therefore, the possibility distribution of a given parameter $u$ quantifies the possible values that this parameter can assume. Differently, a probability distribution quantifies the chances of the parameter $u$ to assume a certain value $x$.

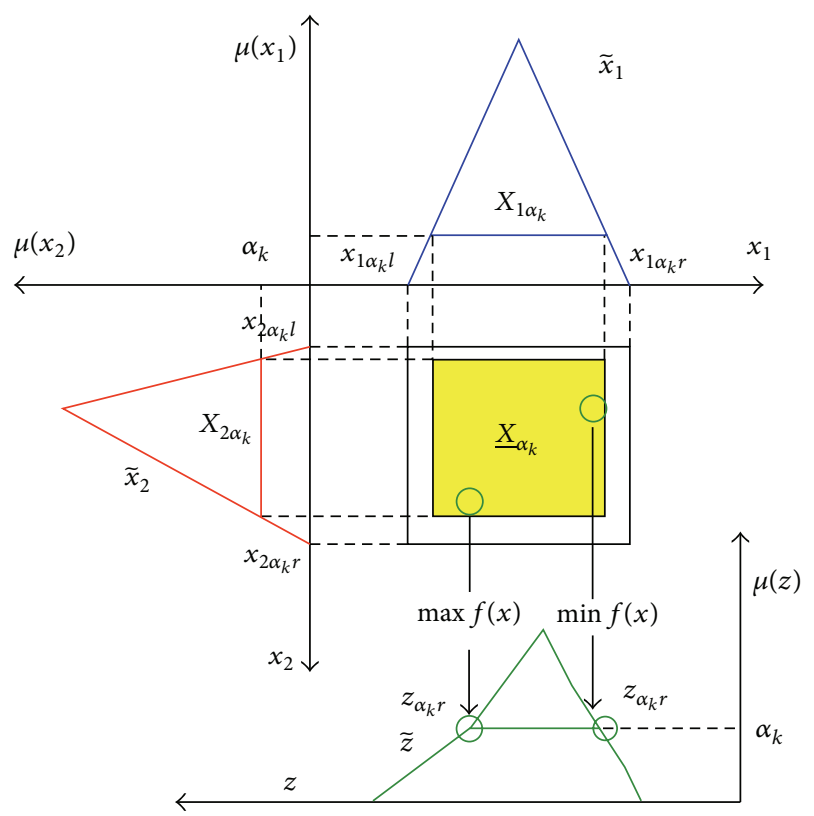

FIGURE 1: The $\alpha$-level optimization.

In the fuzzy set theory, the uncertainties are described as fuzzy inputs and the mathematical model is represented as a fuzzy function that maps those inputs. The extension principle [23] is a widely known method for mapping fuzzy inputs, in which the mapping process is made through the evaluation of an associated mathematical function. Another method used for mapping the fuzzy inputs is the so-called $\alpha$-level optimization [11]. In this case, the fuzzy inputs are discretized by means of $\alpha$-cuts, thus creating a crisp subspace that is the search space of an associated optimization problem. The optimization process is carried out in order to find the minimum and maximum values of the model output for each $\alpha$-level (i.e., lower and upper limits of the correspondent $\alpha$-level, resp.; see Figure 1). The $\alpha$-level optimization is an eficient methodology for mapping fuzzy inputs. However, the effectiveness of the method is highlly related to the performance of the selected optimization technique.

The mapping of a fuzzy input generates an associated fuzzy output. Considering a decision making system, the fuzzy output should be defuzzified into a single value (i.e., a crisp value) that represents the uncertain information. Therefore, the defuzzification is the process of aggregation of the fuzzy information in a single value [24] that can be used to generate robust data.

Different defuzzification techniques exist in the literature to obtain robust data, as follows. The largest of the maximum method selects as crisp value the largest value among all realizations of the fuzzy number. The smallest of the maximum method chooses from the maximum membership function values the smallest one as crisp value. The mean of maximum method takes as crisp value the one obtained from the mean value between the smallest and largest maxima. However, the most commonly used defuzzification techniques are the so-called centroid and bisection methods [25]. The centroid 


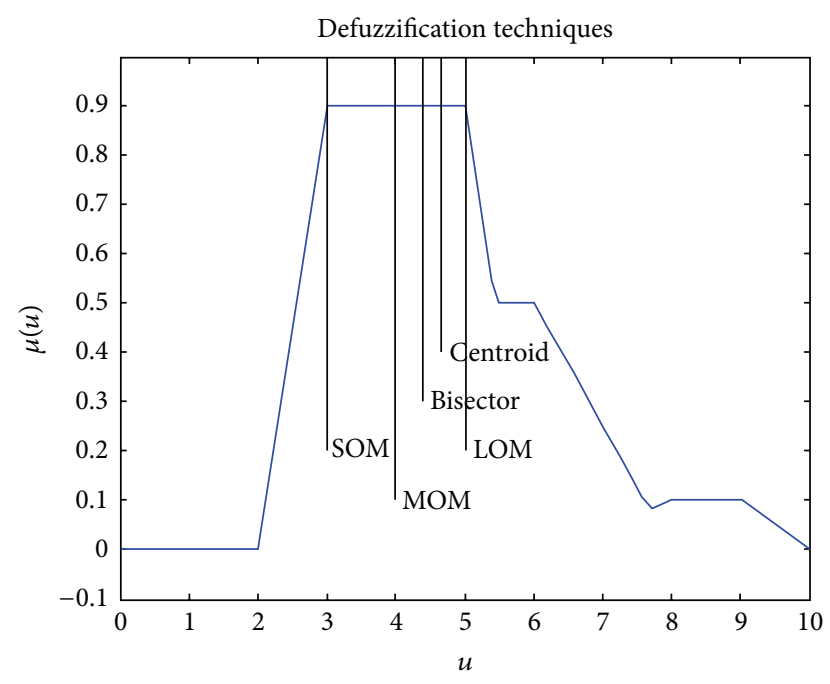

Figure 2: Defuzzification techniques.

method determines the robust value from the area center (gravity center) of the membership function. Similarly, the bisection method calculates the bisector of the fuzzy output to obtain the crisp value. Figure 2 illustrates the presented defuzzification techniques. These aspects are the key points of fuzzy logic and fuzzy uncertainty analysis.

\section{Fuzzy Robust Optimization}

In general, robust optimization is performed relying on the stochastic theory, where a set of samples of the design variables (i.e., a population) is generated and robustness is evaluated by means of the statistical analysis of this set. The stochastic theory is an efficient robust optimization approach, especially when associated with heuristic optimization algorithms (genetic algorithms or differential evolution [26, 27]) and sampling methods such as the Latin Hypercube [28]. However, this procedure usually creates a large combinatorial problem imposing high computational costs.

The fuzzy uncertainty analysis can be used as an alternative approach to reduce the mentioned computational cost and, consequently, to increase the efficiency of the robust optimization process. Fuzzy uncertainty analysis through the $\alpha$-level optimization requires fewer evaluations of the cost function, presenting lower computational cost as compared with the stochastic approach. The associated minimization and maximization processes can be performed by using direct optimization procedures, such as the SQP (Sequential Quadratic Programing) [29], thus reducing even more the computational effort. Therefore, the development of fuzzy metrics to evaluate the robustness of a given design leads to an efficient tool for robust optimization. In this context, the next section is devoted to the discussion of novel robust optimization methodologies based on the fuzzy approach.

3.1. Dual Optimization. As mentioned, the fuzzy set can be considered as the union of the pessimistic and optimistic values of a given fuzzy variable. Considering the uncertainties

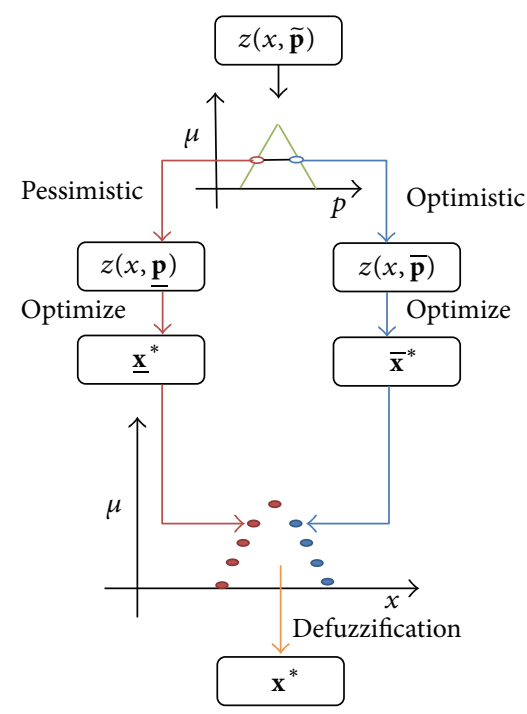

FIGURE 3: Dual optimization procedure flowchart.

of a model as fuzzy variables, a robust optimum should take into account the possible realizations of the uncertain values. In this context, a robust optimization procedure has to consider the pessimistic and optimistic values of the uncertain parameters during the optimization tasks.

A dual optimization problem can be conceived by treating the optimization problem as a fuzzy function and following a methodology similar to the $\alpha$-level optimization. The pessimistic and optimistic boundaries (i.e., the lower and upper bounds, resp.) associated with the uncertain parameters are created for each $\alpha$-level. A fuzzy optimum is then obtained considering two different optimization problems: (i) an optimization problem where the goal is to find an optimum by considering the pessimistic values of the uncertain information for the related $\alpha$-level and (ii) a second optimization problem dedicated to searching an optimum by taking into account the optimistic values for the same $\alpha$-level. This fuzzy optimum is a set of possible optima, that is, a fuzzy information that can be aggregated into a robust optimum through the defuzzification process. The dual optimization procedure is illustrated in Figure 3.

Defining $\mathbf{x}$ as the vector of deterministic design variables and $\widetilde{\mathbf{p}}$ as the vector of uncertain values, a pseudo-objective function (sequential unconstrained minimization technique [29]) is expressed as

$$
\Phi(\mathbf{x}, \widetilde{\mathbf{p}}, r p)=F(\mathbf{x}, \widetilde{\mathbf{p}})+r p * P(\mathbf{x}, \widetilde{\mathbf{p}})
$$

where $r p$ is the vector of the penalty factors related with the penalty functions, $F$ is the vector of the original cost function, $P$ is the vector of constraints, and $\Phi$ is the resulting pseudoobjective function. 
Based on the possibility theory, the dual optimization problem can be formulated as presented in (2) and (3). Consider the following:

$$
\begin{aligned}
& \Phi(\mathbf{x}, \underline{\mathbf{p}}, r p)=\underline{F}(\mathbf{x}, \underline{\mathbf{p}})+r p * \underline{P}(\mathbf{x}, \underline{p}), \\
& \Phi(\mathbf{x}, \overline{\mathbf{p}}, r p)=\bar{F}(\mathbf{x}, \overline{\mathbf{p}})+r p * \bar{P}(\mathbf{x}, \overline{\mathbf{p}})
\end{aligned}
$$

in which $\underline{\mathbf{p}}$ and $\overline{\mathbf{p}}$ represent the lower and upper bounds of the uncertain values, respectively, $\underline{F}$ and $\bar{F}$ are the lower and upper bounds of the cost functions vector, $\underline{P}$ and $\bar{P}$ are the bounderies of the constraints vector, and $\underline{\Phi}$ and $\bar{\Phi}$ are the pessimistic and optimistic pseudo-objective functions, respectively.

The optimum for the optimization problem expressed by (2) leads to a lower bound optimal value $\underline{\mathbf{x}}^{*}$, while the optimization problem described by (3) results in an upper bound optimal value $\overline{\mathbf{x}}^{*}$. Then, at each $\alpha$-level two sets of optimal results are generated, namely, the lower optima and the upper optima (i.e., pessimistic and optimistic values, resp.). The union of the two sets generates a fuzzy set or a fuzzy optimum, which possibly contain the optimal solutions of the design problem. This fuzzy information can be addressed to a robust solution through a defuzzification procedure, as previouslly mentioned.

3.2. Amplitude Minimization. In this case, it is assumed that the robustness metric can be defined regarding the amplitude of the envelope determined by the cost functions given by (2) and (3). The higher the amplitude of the obtained envelope (i.e., the difference between the optimistic and pessimistic responses) the lower the robustness of the solution. Consequently, a more robust solution will present envelopes with lower amplitude (i.e., smaller difference between the optimistic and pessimistic responses). Thus, the robustness metric that measures the distance between the optimistic and pessimistic responses (measure of the response amplitude) is now defined. Equation (4) presents the proposed robustness metric for the amplitude minimization scheme:

$$
r o_{i}=\sqrt{\left(\bar{\Phi}_{i}(\mathbf{x}, \overline{\mathbf{p}}, r p)-\underline{\Phi}_{i}(\mathbf{x}, \underline{\mathbf{p}}, r p)\right)^{2}} \quad i=1, \ldots, n,
$$

where $n$ is the total number of points in which the pseudoobjective functions are evaluated. The distance between the optimistic and the pessimistic responses is then computed by using (4).

Therefore, a new objective function $z$ (5) is formulated to find the solution associated with the highest robustness value that minimizes the proposed metric (4). Figure 4 summarizes the amplitude minimization procedure

$$
z(\mathbf{x}, \widetilde{\mathbf{p}}, r p)=\min \left(\sum_{i=1}^{n} r o_{i}\right) .
$$

3.3. Fuzzy Response Minimization. In the case of a robust optimization problem (considering uncertainties), for a given design (the configuration of the design variables $\mathbf{x}$ ) a fuzzy

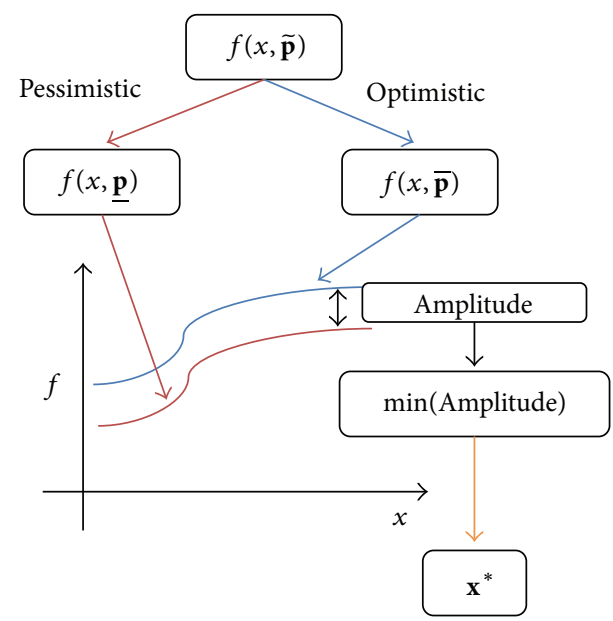

FIgURE 4: Flowchart of the amplitude minimization procedure.

uncertainty analysis is performed generating a fuzzy output. As previously mentioned, this fuzzy output is the union of the pessimistic and optimistic responses, in which the $\alpha$ level boundaries represent the lower and the upper bounds of all possible outcomes of the optimization problem. Consequently, it can be inferred that a robust solution will have the smallest difference between these bounds and therefore the smallest area for its fuzzy output.

Thus, in the fuzzy response minimization scheme, the robust optimization procedure is formulated aiming at finding the robust optimum, which is represented by the samallest area of the fuzzy output (see Figure 1). Defining $Z$ as the function that represents the optimization problem, the uncertainty analysis can be defined by

$$
Z(\mathbf{x}, \widetilde{\mathbf{p}})=Z(\mathbf{x}, f(\alpha)) .
$$

The area of the fuzzy output can be viewed as the robustness metric wherein its minimization leads to a robust optimum. Therefore, the metric is calculed from (7). Figure 5 illustrates the main concepts of the fuzzy response minimization procedure

$$
\begin{aligned}
\text { Area } & =\int_{0}^{1} Z(\mathbf{x}, f(\alpha)) d \alpha \\
& =\int_{0}^{1} \underline{Z}(\mathbf{x}, \underline{f}(\alpha)) d \alpha+\int_{0}^{1} \bar{Z}(\mathbf{x}, \bar{f}(\alpha)) d \alpha .
\end{aligned}
$$

All the robust optimization procedures conceived in this contribution are formulated as single-objective optimization problems in which the objective function is directly related to the robustness of the problem. However, all these procedures can be easily extended to multiobjective optimization problems by considering a cost function related to the original optimization problem and another one related to the robustness evaluation (one of the proposed criteria).

In order to model an uncertain quantity, the perspective of the degree of uncertainty is very interesting. However, the degree of preference seems to be more appropriate for 


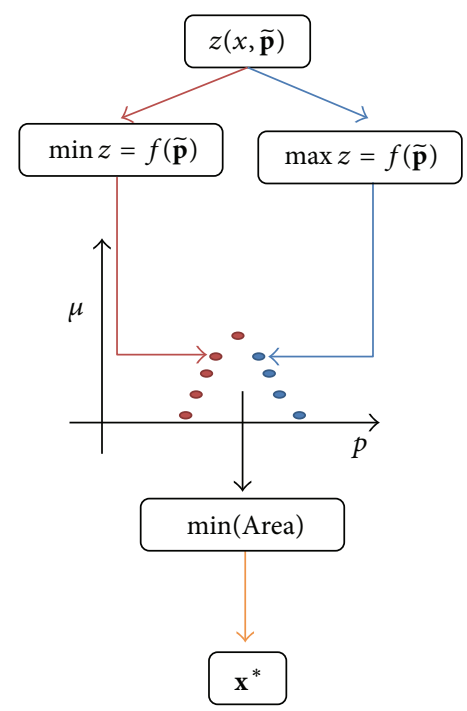

Figure 5: Flowchart for the fuzzy response minimization procedure.

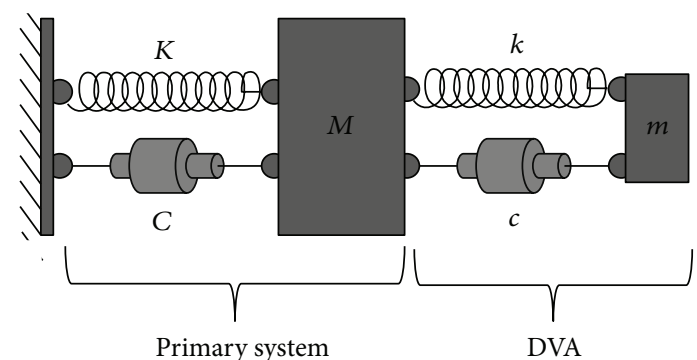

Figure 6: Classical DVA scheme.

optimization purposes. Nevertheless, in the fuzzy robust optimization criteria presented in this paper, the interpretation of the degree of uncertainty is used both to model the uncertain parameters (fuzzy variables) and to define the optimization task.

In addition, the centroid method was used for defuzzification, once this method is the closest to what can be defined as the weighted average of a fuzzy set.

\section{Dynamic Vibration Absorbers: A Review}

4.1. Linear Vibration Absorber (DVA). A linear dynamic vibration absorber (DVA) is a mechanical system composed by inertia, stiffness, and damping elements, which is attached to a primary system aiming at attenuating the vibration response [31]. The classical one-degree-of-freedom DVA coupled to the primary system is presented by Figure 6 .

The vibration amplitude of the primary system vanishes when the natural frequency of the DVA matches the excitation frequency of the force acting on the primary system [31]. The DVA is tuned by properly choosing its mechanical properties, so that the vibration energy of the primary system can be mitigated. Equation (8) presents the dimensionless vibration amplitude of the mechanical system presented in Figure 6. Consider

$$
\frac{\left|X_{p}\right|}{\left(X_{p}\right)_{s t}}=\sqrt{\left\{\frac{(2 \zeta g)^{2}+\left(g^{2}-f^{2}\right)^{2}}{(2 \zeta g)^{2}\left(g^{2}-1+\mu g^{2}\right)^{2}+\left[\mu f^{2} g^{2}-\left(g^{2}-1\right)\left(g^{2}-f^{2}\right)^{2}\right]^{2}}\right\}},
$$

where the following parameters are considered: $\mu=m_{a} / m_{p}$ is the mass ratio, $f=\omega_{a} / \omega_{p}$ is the turning factor, $g=\omega / \omega_{p}$ is the forcing frequency ratio, $c_{c}=2 m_{a} \omega_{p}$ is the critical damping, $\zeta=c_{a} / c_{c}$ is the damping ratio, $\left(X_{p}\right)_{\text {st }}=F_{0} / k_{p}$ is the static displacement of the primary mass, and $X_{p}$ is the displacement of the primary mass. In this case, $m_{p}, c_{p}$, and 
$k_{p}$ are the mass, damping factor, and stiffness coefficient of the primary system, respectively. For the DVA, $m_{a}, c_{a}$, and $k_{a}$ are the mass, damping factor, and stiffness coefficient; $\omega$ is the frequency of the external force, $\omega_{a}=\sqrt{ }\left(k_{a} / m_{a}\right)$ is the natural frequency of the DVA, $\omega_{p}=\sqrt{ }\left(k_{p} / m_{p}\right)$ is the natural frequency of the primary system, and $F_{0}$ is the amplitude of the external force.

Mathematically, the optimum design of a DVA consists in determining the parameters $f$ and $\zeta$ that minimize the maximum amplitude of the frequency response function of the complete system described by (8) (i.e., the primary system plus the DVA; see Figure 6).

4.2. Nonlinear Dynamic Vibration Absorber ( $n D V A)$. The primary system, the DVA, or both systems may contain nonlinear elements (particularly the springs) that exhibit nonlinear behavior. In [30] the authors analyzed the limitations and capabilities of a nonlinear dynamic vibration absorber (nDVA). In this work, the robust design of a one-degree-offreedom nDVA was carried out by taking into account the response of the system as described in the frequency domain.

Equations (9) present a system of algebraic equations representing the dynamic behavior of an nDVA coupled to a nonlinear primary system (stiffness nonlinearity). This mathematical formulation was adopted in [30], in which the Van der Pol Transformation was used. Consider

$$
\begin{aligned}
& \left(1+\mu-\omega_{p}^{2}\right) u_{p}+\mu u_{a}-2 \zeta_{p} \omega_{p} v_{p}-\frac{3}{4} \zeta_{p} u_{p}\left(u_{p}^{2}+v_{p}^{2}\right) \\
& \quad+F=0, \\
& \mu u_{p}+\left(\mu-\mu \omega_{a}^{2}\right) u_{a}-\mu 2 \zeta_{a} \omega_{a} v_{a} \\
& \quad-\frac{3}{4} \mu \zeta_{a} u_{a}\left(u_{a}^{2}+v_{a}^{2}\right)=0, \\
& \left(\omega_{p}^{2}-1-\mu\right) v_{p}+\mu v_{a}-2 \zeta_{p} \omega_{p} u_{p} \\
& \quad+\frac{3}{4} \zeta_{p} v_{p}\left(u_{p}^{2}+v_{p}^{2}\right)=0, \\
& \mu v_{p}+\left(\mu-\mu \omega_{a}^{2}\right) v_{a}-\mu 2 \zeta_{a} \omega_{a} u_{a} \\
& \quad-\frac{3}{4} \mu \zeta_{a} v_{a}\left(u_{a}^{2}+v_{a}^{2}\right)=0,
\end{aligned}
$$

where the displacements $u$ and velocities $v$ of the primary system $(p)$ and nDVA $(a)$ (vibration responses $u_{p}, u_{a}, v_{p}$, and $v_{a}$, resp.) are assumed to be slow functions of a normalized time. In this case, the formulation of new parameters is also required, as follows: $\zeta_{i}=k_{i}^{n l} x_{c}^{2} /\left(m_{i} \omega^{2}\right)$, where $x_{c}=F_{0} / k_{p}$ and $k_{i}^{n l}$ is the nonlinear stiffness coefficient of the spring $(i=p, a)$, and $F=F_{0} /\left(m_{p} x_{c} \omega^{2}\right)$. The solution of this algebraic system is obtained by defining the parameters $\beta=F / \omega_{p}^{2}, \Omega=\omega / \omega_{p}$, so that in the algebraic system one has $\omega_{p}=1 / \Omega$ and $\omega_{a}=\rho / \Omega$, where $\rho=\omega_{a} / \omega_{p}$.

For the optimum design of the nDVA, a multiobjective optimization problem is formulated considering a vector

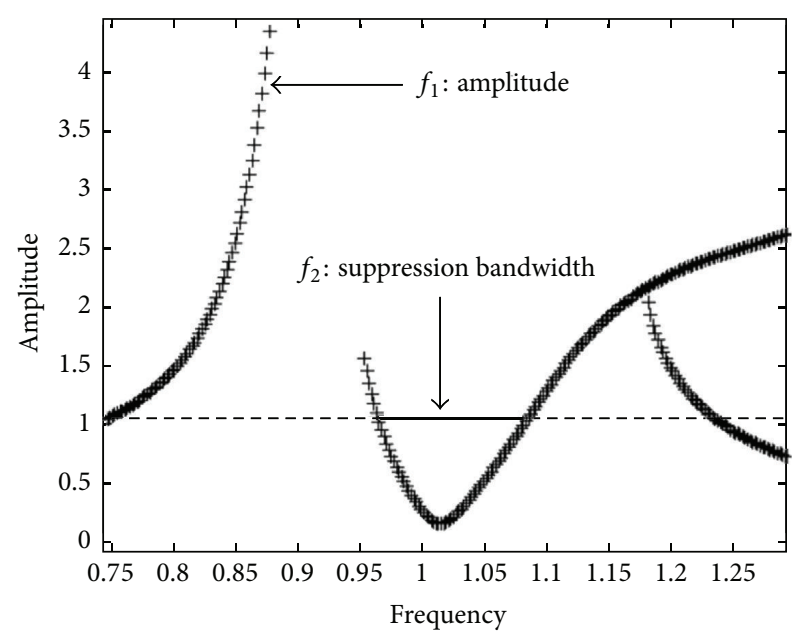

FIGURE 7: Objective functions of the nDVA design problem (adapted from [30]).

containing two objective functions. The first is devoted to the minimization of the vibration amplitude associated with the first vibration mode of the nonlinear damped system. The second objective function of the problem is related to the maximization of the suppression bandwidth, as achieved by the nDVA. Figure 7 illustrates the two objective functions of the considered optimization process.

\section{Numerical Results}

In this paper, the proposed fuzzy robust optimization techniques were applied to the design of the systems depicted in Figure 8 (DVA, Figure 8(a), and nDVA, Figure 8(b)). Regarding the DVA design, uncertainties affecting its stiffness $k_{a}$ and damping $c_{a}$ coefficients were taken into account. For the design of the nDVA, the parameters $\xi_{a}, \mu, \beta$, and $\rho$ were considered uncertain.

Considering the robust design of the DVA, the uncertain parameters were modeled as fuzzy triangular numbers with an uncertainty of $\pm 10 \%$ with respect to the nominal value of the parameter. Table 1 shows the uncertainty scenario considered in the present contribution. In the design of the nDVA, the uncertain parameters were also treated as fuzzy triangular number. However, an uncertainty range of $\pm 30 \%$ was adopted with respect to the nominal values. Table 2 shows the uncertainty scenario considered for the nDVA design. Additionally, the masses for the primary and DVA subsystems are also given. It is worth mentioning that for the design of the DVA, the uncertainty associated with the stiffness of the primary system $k_{p}$ also affects the parameters $f, \zeta$, and the forcing frequency factor $g$ (see (8)). In the following, the masses adopted for the DVA were the same as those used for the nDVA.

The simulation of the DVA was performed considering a frequency bandwidth of $5 \mathrm{~Hz}$ (i.e., $\omega=5-10 \mathrm{~Hz}$ ), with a sample frequency of $0.001 \mathrm{~Hz}$. The design of the nDVA was carried out by taking into account a frequency bandwidth of $0.6 \mathrm{~Hz}$ (i.e., $\Omega=0.7-1.3 \mathrm{~Hz}$ ), comprising 300 frequency steps. 
TABLE 1: Parameters used for the design of the linear DVA.

\begin{tabular}{|c|c|c|c|c|c|c|c|}
\hline \multirow{2}{*}{$m_{p}[\mathrm{~kg}]$} & \multirow{2}{*}{$m_{a}[\mathrm{~kg}]$} & \multicolumn{3}{|c|}{$k_{p}[\mathrm{~N} / \mathrm{m}]$} & \multicolumn{3}{|c|}{$F_{0}[\mathrm{~N}]$} \\
\hline & & Lower* & Nominal & Upper* & Lower* & Nominal & Upper $^{*}$ \\
\hline 100 & 2 & 4500 & 5000 & 5500 & 0.9 & 1 & 1.1 \\
\hline
\end{tabular}

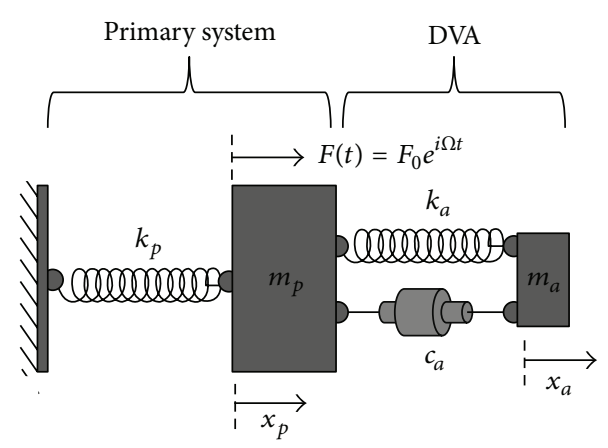

(a)

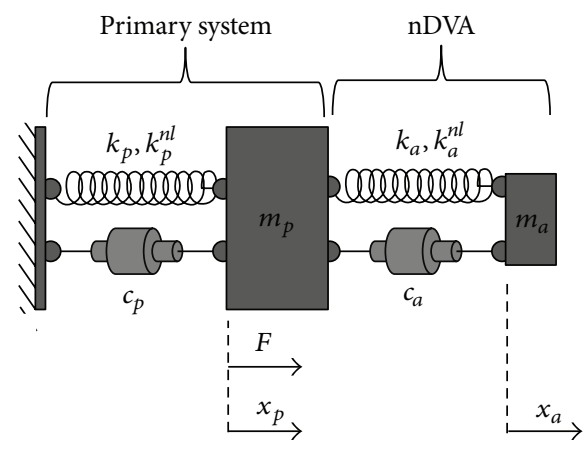

(b)

FIGURE 8: Linear (DVA) and nonlinear (nDVA) dynamic vibration absorbers.

TABle 2: Parameters used for the design of the nDVA.

\begin{tabular}{lccccccc}
\hline Parameters & $\xi_{p}$ & $\xi_{a}$ & $\zeta_{p}$ & $\zeta_{a}$ & $\mu$ & $B$ & $\rho$ \\
\hline Nominal & 0.0001 & 0.01 & 0.01 & 0.01 & 0.05 & 0.1 & 1.0 \\
Lower $^{*}$ & - & 0.007 & - & - & 0.035 & 0.07 & 0.7 \\
Upper $^{*}$ & - & 0.013 & - & - & 0.065 & 0.13 & 1.3 \\
\hline
\end{tabular}

${ }^{*}$ Lower stands for lower limit. Upper stands for upper limit.

TABLE 3: Optimization results obtained by the DVA design problem.

\begin{tabular}{lcccc}
\hline Approach & $k_{a}[\mathrm{~N} / \mathrm{m}]$ & $c_{a}[\mathrm{Ns} / \mathrm{m}]$ & Cost function & Robustness \\
\hline Deterministic & 96.2744 & 1.9550 & 0.0000 & 24.2396 \\
Dual optm. & 96.2371 & 2.0474 & 0.0020 & 23.4711 \\
Amplitude min & 94.6159 & 2.1958 & 1.7131 & 22.0145 \\
Fuzzy response & 95.4366 & 2.1794 & 0.8235 & 22.2918 \\
\hline
\end{tabular}

Regarding the solution of the algebraic system defined by (9), the initial conditions of the nDVA were considered as follows: $u_{p}=5 \mathrm{~m}, u_{a}=5 \mathrm{~m}, v_{p}=2 \mathrm{~m} / \mathrm{s}$, and $v_{a}=4 \mathrm{~m} / \mathrm{s}$. The DVA was considered at rest for $t=0$.

Table 3 presents the obtained results applying both the deterministic and robust optimization procedures related to the design of the DVA. The robustness metrics, presented in (4), were used to evaluate the performance of the proposed fuzzy techniques. Note that using the amplitude minimization procedure the smallest robust parameter resulted, which is similar to the one determined for the case in which the fuzzy response minimization was applied. Additionally, it can be observed that the dual optimization procedure led to the parameters with the lowest associated robustness. Regarding the cost function (i.e., the difference between the invariant points), the amplitude minimization procedure led to the worst results. The envelopes obtained for each robust procedure proposed in this work are presented in Figure 9.
Table 4 presents the results (taken from [30]) obtained considering the deterministic and robust optimization problem related to the design of the nDVA. The design of the nDVA is a multiobjective optimization problem that was solved by using the compromise programming technique [29] considering equal weight cost functions (i.e., the same weighting factor for both cost functions). The targets of the optimization problem were the results obtained by analyzing each cost function separately. The worst known value for each objective function was adopted as the value of each cost function evaluated at the initial point. Therefore, the multiobjective problem was handled as a representative monoobjective optimization problem, resulting in a single optimum instead of a Pareto frontier.

As observed in the DVA problem, the amplitude minimization technique presented the most robust optimum value, followed by the fuzzy response minimization procedure. In this case, the differences between the robustness metrics were most likely caused by numerical approximations. The dual optimization approach led to the worst result. In contrast with the design of the linear system, the robustness of the nDVA increases significantly by applying the proposed fuzzy techniques as compared with the deterministic solution. Concerning the cost functions (minimization of $F_{1}$ and maximization of $F_{2}$; see Table 4 ), all the proposed fuzzy methodologies presented better solutions than the ones (taken from [30]) obtained from the deterministic approach. Figure 10 presents the envelopes obtained for each proposed fuzzy approach for the design of the nDVA.

\section{Concluding Remarks}

In this paper, three robust optimization methodologies based on the fuzzy logic approach were proposed and evaluated for the robust design of linear and nonlinear dynamic vibration 
TABLE 4: Optimization results obtained by the nDVA design problem.

\begin{tabular}{lccccccc}
\hline Approach & $\xi_{a}$ & $\mu$ & $\beta$ & $\rho$ & $F_{1}$ & $F_{2}$ & Robustness \\
\hline Deterministic & 0.0092 & 0.0550 & 0.090 & 1.1000 & 8.2487 & 0.1043 & 245.90 \\
Dual optm. & 0.0078 & 0.0402 & 0.0780 & 1.1982 & 3.7370 & 0.1365 & 172.87 \\
Amplitude min & 0.0070 & 0.0350 & 0.0700 & 1.2993 & 3.4338 & 0.1485 & 142.42 \\
Fuzzy response & 0.0071 & 0.0350 & 0.0700 & 1.2996 & 3.4345 & 0.1485 & 143.51 \\
\hline
\end{tabular}
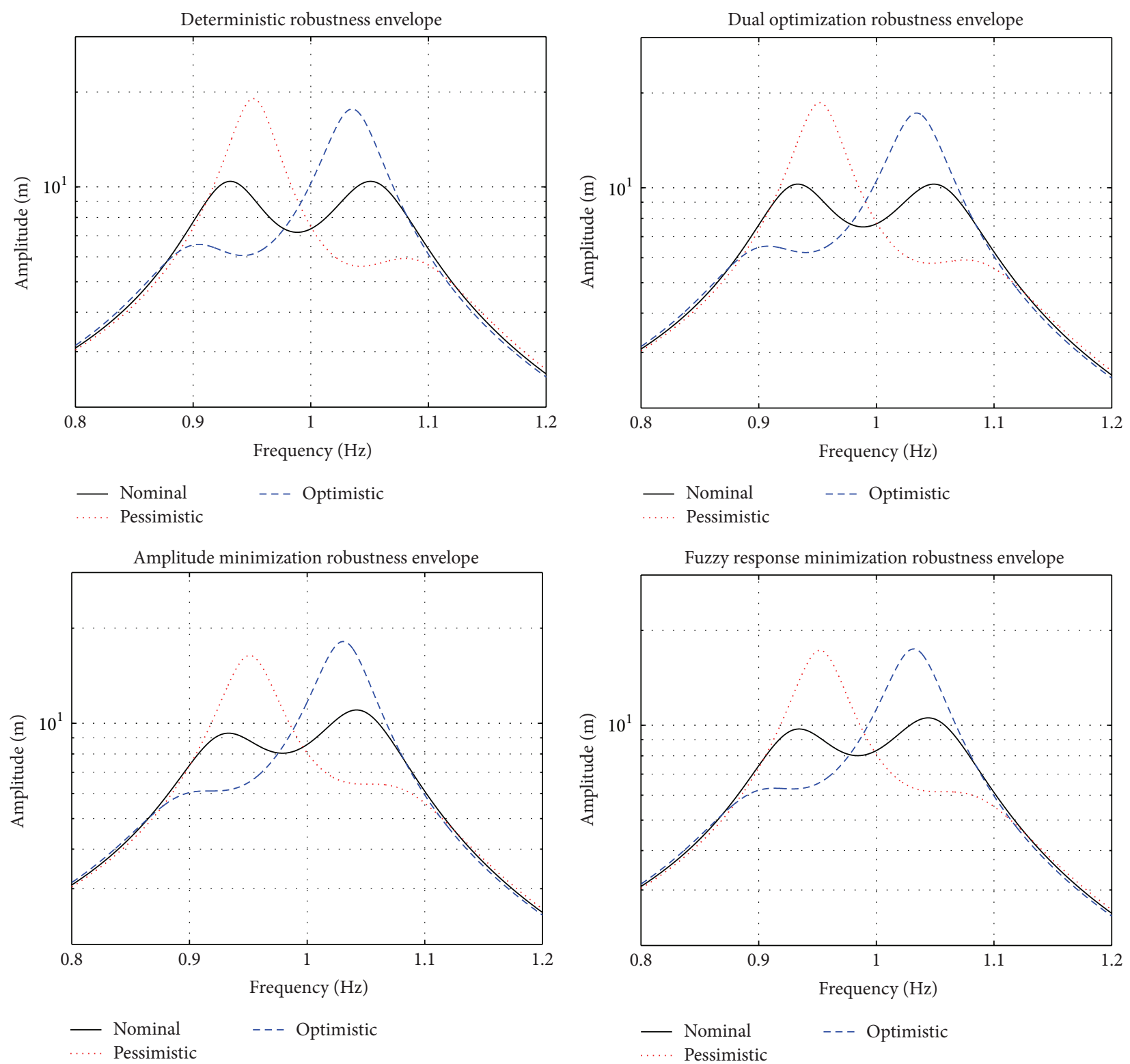

FIGURE 9: Robustness envelopes determined in the design of the DVA.

absorbers. The originality regarding the robust optimization methodology is related to the formulation and use of fuzzy metrics to evaluate the design robustness, handling the robust optimization problem as a monoobjective optimization instead of imposing a new cost function to the design problem and thus leading to a multiobjective optimization problem.

The results demonstrated that, for all the considered design problems, the proposed methodologies found solutions that are more robust than the ones obtained from 

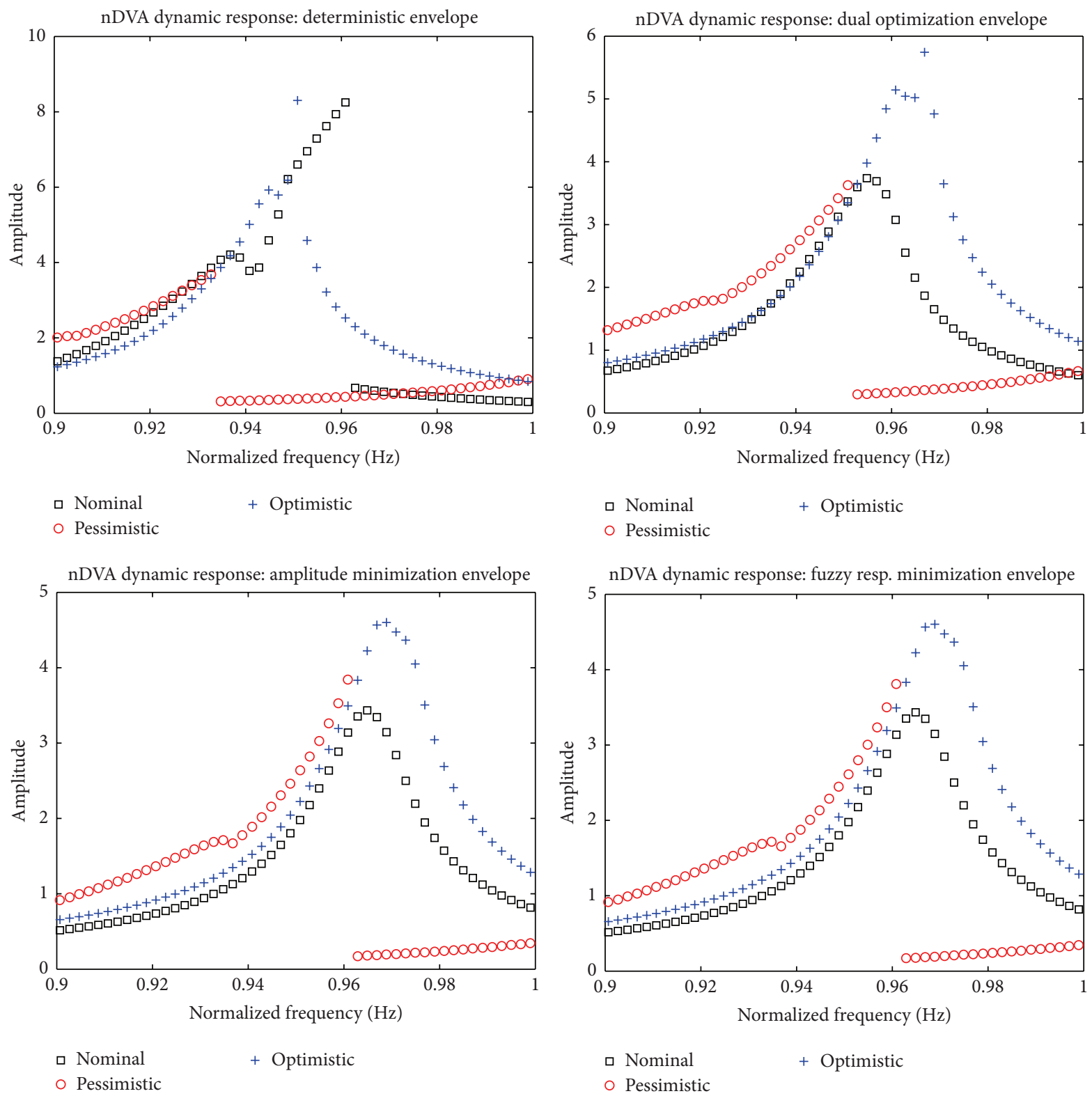

FIGURE 10: Robustness envelopes determined in the design of the DVA.

the deterministic approach. Additionally, the so-called amplitude minimization procedure obtained the most robust solution for all considered design problems.

Finally, the methodologies conveyed demonstrate that fuzzy optimization techniques are viable for robust optimization problems and may even be more suitable for this kind of design problem as compared with other competing alternatives, due to its mathematical simplicity and less number of evaluations of the objective function.

\section{Conflict of Interests}

The authors declare that there is no conflict of interests regarding the publication of this paper.

\section{References}

[1] L. A. Zadeh, "Fuzzy sets," Information and Control, vol. 8, pp. 338-353, 1965.

[2] L. A. Zadeh, "Fuzzy sets as a basis for a theory of possibility," Fuzzy Sets and Systems, vol. 1, no. 1, pp. 3-28, 1978.

[3] R. G. Ghanem and P. D. Spanos, Stochastic Finite Elements: a Spectral Approach, Springer, New York, NY, USA, 1991.

[4] J. Zhang and B. Ellingwood, "Orthogonal series expansions of random fields in reliability analysis," Journal of Engineering Mechanics, vol. 120, no. 12, pp. 2660-2677, 1994.

[5] L. A. Hannah, "Stochastic optimization," International Encyclopedia of the Social \& Behavioral Sciences, vol. 2, pp. 473-481, 2015 . 
[6] C. Mares, J. E. Mottershead, and M. I. Friswell, "Stochastic model updating: part I-theory and simulated example," Mechanical Systems and Signal Processing, vol. 20, pp. 1674-1695, 2006.

[7] J. Didier, B. Faverjon, and J.-J. Sinou, "Analysing the dynamic response of a rotor system under uncertain parameters by polynomial chaos expansion," Journal of Vibration and Control, vol. 18, no. 5, pp. 712-732, 2012.

[8] E. H. Koroishi, A. A. Cavalini Jr., A. M. G. de Lima, and V. Steffen Jr., "Stochastics modeling of flexible rotors," Journal of the Brazilian Society of Mechanical Sciences and Engineering, vol. 34, pp. 597-603, 2012.

[9] E. Mrabet, M. Guedri, M. N. Ichchou, and S. Ghanmi, "Stochastic structural and reliability based optimization of tuned mass damper," Mechanical Systems and Signal Processing, vol. 60-61, pp. 437-451, 2015.

[10] M. Carrasco, B. Ivorra, and A. M. Ramos, "Stochastic topology design optimization for continuous elastic materials," Computer Methods in Applied Mechanics and Engineering, vol. 289, pp. 131154, 2015.

[11] B. Möller, W. Graf, and M. Beer, "Fuzzy structural analysis using $\alpha$-level optimization," Computational Mechanics, vol. 26, no. 6, pp. 547-565, 2000.

[12] G. C. Marano and G. Quaranta, "Fuzzy-based robust structural optimization," International Journal of Solids and Structures, vol. 45, no. 11-12, pp. 3544-3557, 2008.

[13] A. A. Cavalini Jr., F. A. Lara-Molina, A. D. G. Silva, and V. Steffen Jr., "Experimental uncertainty analysis of a flexible rotor supported by fluid film bearings," in Proceedings of the International Symposium on Dynamic Problems of Mechanics (DINAME '15), Natal, Brazil, 2015.

[14] W. Graf, M. Götz, and M. Kaliske, "Analysis of dynamical processes under consideration of polymorphic uncertainty," Structural Safety, vol. 52, pp. 194-201, 2015.

[15] G. Taguchi, Quality Engineering through Design Optimization, Kraus International Publications, 1984.

[16] I. Paenke, J. Branke, and Y. Jin, "Efficient search for robust solutions by means of evolutionary algorithms and fitness approximation," IEEE Transactions on Evolutionary Computation, vol. 10, no. 4, pp. 405-420, 2006.

[17] H.-G. Beyer and B. Sendhoff, "Robust optimization-a comprehensive survey," Computer Methods in Applied Mechanics and Engineering, vol. 196, no. 33-34, pp. 3190-3218, 2007.

[18] A. Soroudi and T. Amraee, "Decision making under uncertainty in energy systems: state of the Art," Renewable and Sustainable Energy Reviews, vol. 28, pp. 376-384, 2013.

[19] V. Gabrel, C. Murat, and A. Thiele, "Recent advances in robust optimization: an overview," European Journal of Operational Research, vol. 235, no. 3, pp. 471-483, 2014.

[20] R. Lima, C. Soize, and R. Sampaio, "Robust design optimization with an uncertain model of a nonlinear vibro-impact electromechanical system," Communications in Nonlinear Science and Numerical Simulation, vol. 23, no. 1-3, pp. 263-273, 2015.

[21] S. Tammareddi, G. Sun, and Q. Li, "Multiobjective robust optimization of coronary stents," Materials and Design, vol. 90, pp. 682-692, 2016.

[22] D. Dubois and H. Prade, "The three semantics of fuzzy sets," Fuzzy Sets and Systems, vol. 90, no. 2, pp. 141-150, 1997.

[23] L. A. Zadeh, "The concept of a linguistic variable and its application to approximate reasoning: part I," Information Sciences, vol. 8, pp. 199-249, 1975.
[24] B. Möller, W. Graf, and M. Beer, Fuzzy Randomness: Uncertainty in Civil Engineering and Computational Mechanics, Springer, 2004.

[25] T. Takagi and M. Sugeno, "Fuzzy identification of systems and its applications to modeling and control," IEEE Transactions on Systems, Man and Cybernetics, vol. 15, no. 1, pp. 116-132, 1985.

[26] N. Srinivas and K. Deb, "Multiobjective using nondominated sorting in genetic algorithms," Tech. Rep., Department of Mechanical Engineering, Institute of Technology, New Delhi, India, 1993.

[27] R. Storn and K. Price, "Differential evolution: a simple and efficient adaptive scheme for global optimization over continuous spaces,' Tech. Rep. TR-95-012, International Computer Science Institute, Berkeley, Calif, USA, 1995.

[28] F. A. C. Viana, G. Venter, V. Balabanov, and V. Steffen Jr., "On how to implement an affordable optimal Latin hypercube," in Proceedings of the 19th Congress of Mechanical Engineering (COBEM '07), Brasília, Brazil, November 2007.

[29] G. N. Vanderplaats, Numerical Optimization Techniques for Engineering Design, Vanderplaats Research and Development, Colorado Springs, Colo, USA, 1999.

[30] R. A. Borges, A. M. G. de Lima, and V. Steffen Jr., "Robust optimal design of a nonlinear dynamic vibration absorber combining sensitivity analysis," Shock and Vibration, vol. 17, no. 4-5, pp. 507-520, 2010.

[31] V. Steffen Jr. and D. A. Rade, "Dynamic vibration absorber," in Encyclopedia of Vibration, pp. 9-26, Academic Press, 2001. 


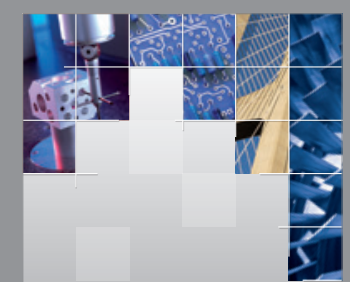

\section{Enfincering}
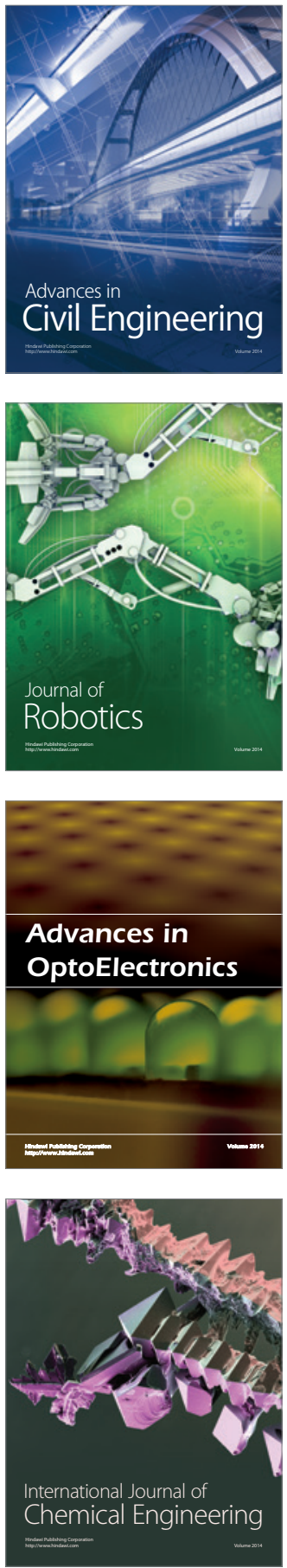

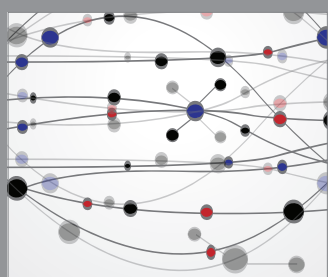

The Scientific World Journal

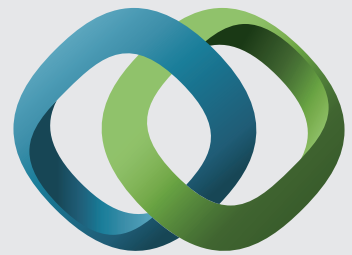

\section{Hindawi}

Submit your manuscripts at

http://www.hindawi.com
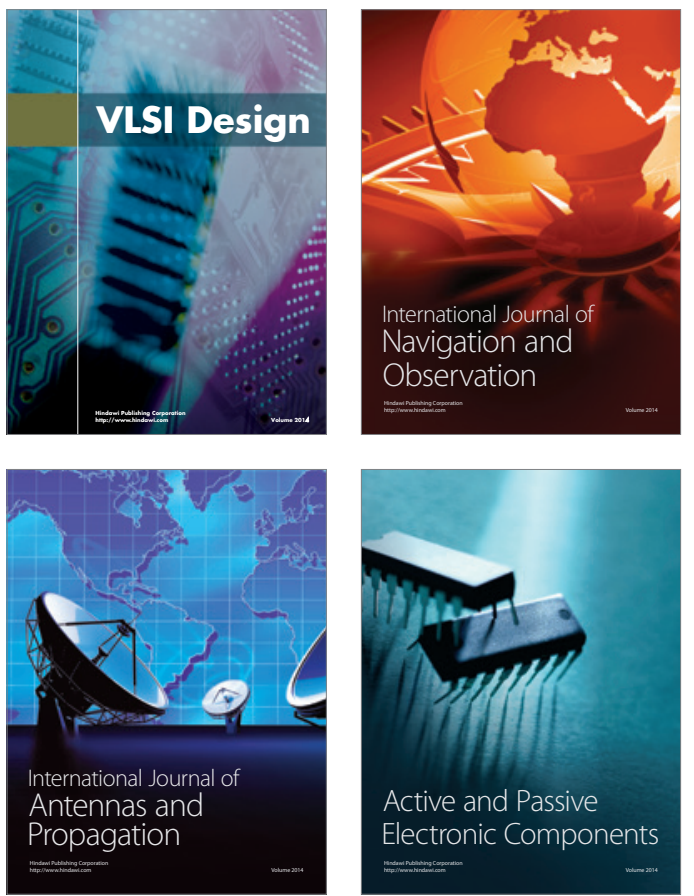
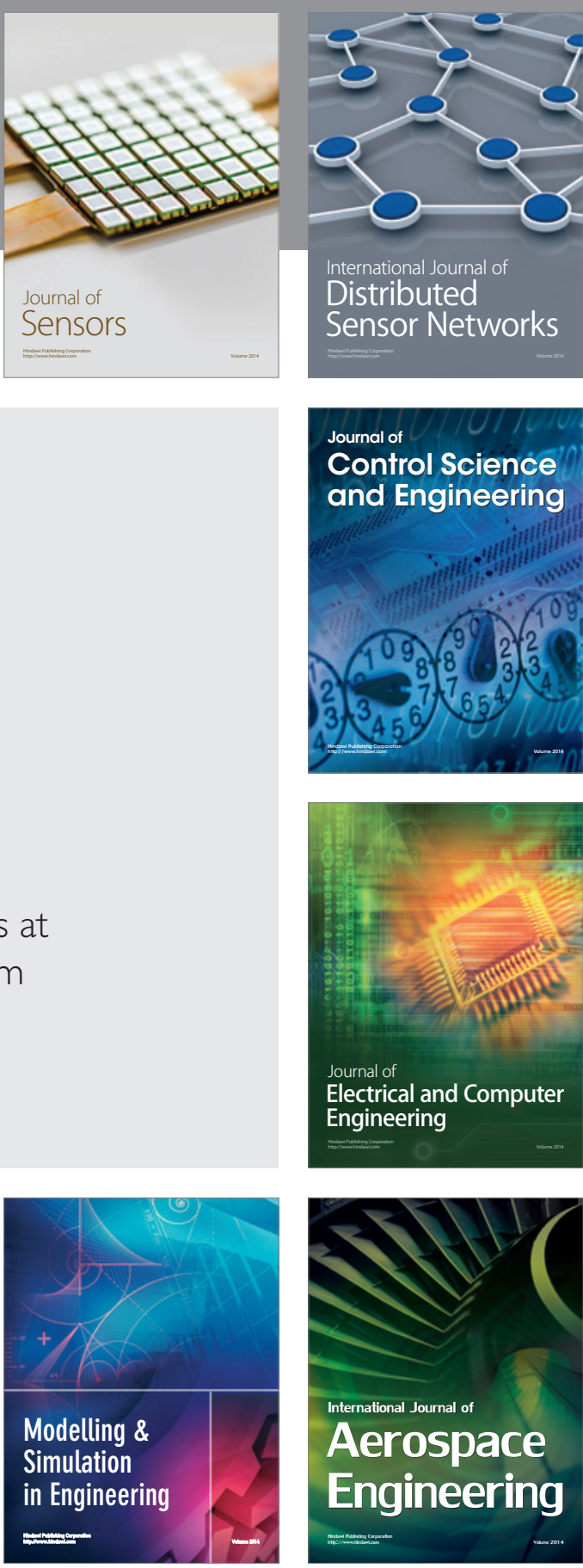

International Journal of

Distributed

Sensor Networks

Journal of

Control Science

and Engineering
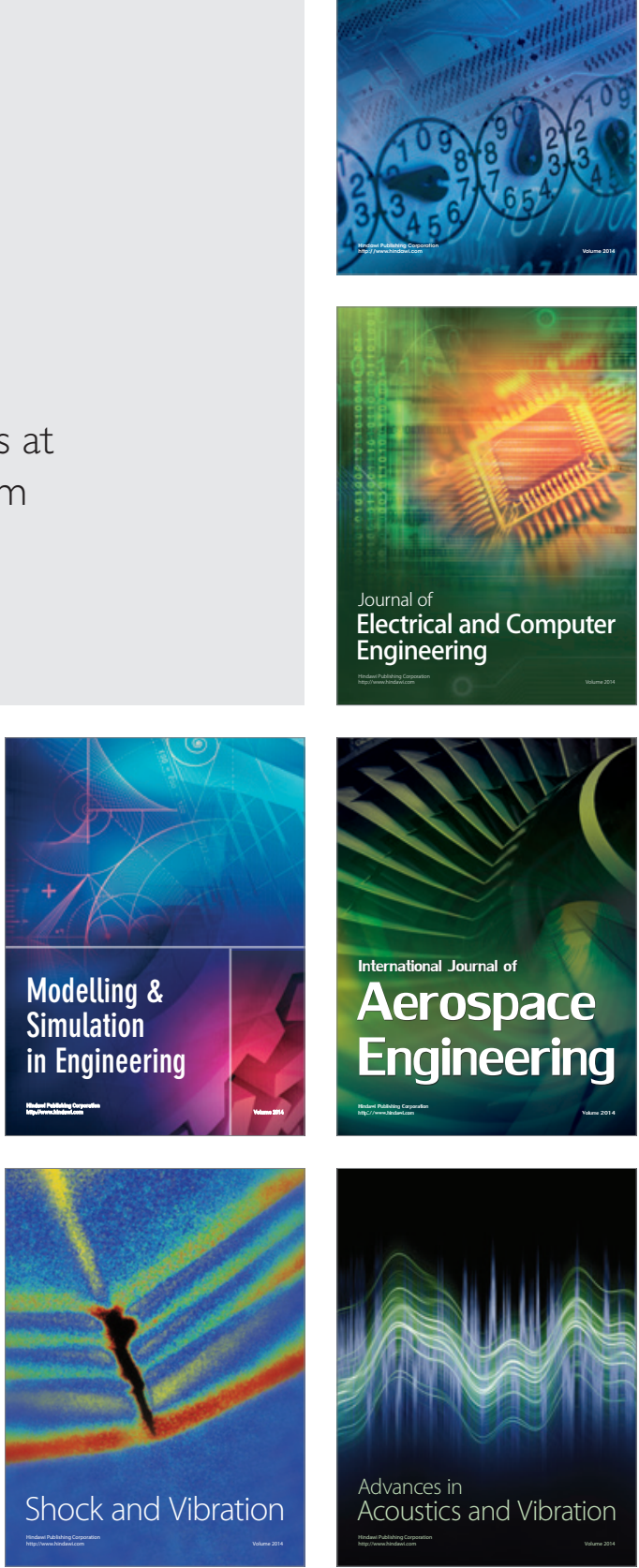